\title{
Trivium
}

Revue franco-allemande de sciences humaines et sociales - Deutsch-französische Zeitschrift für Geistesund Sozialwissenschaften

$5 \mid 2010$

Sociologie des conventions

\section{Werte, Koordination und Rationalität: Die Verbindung dreier Themen durch die "Économie des conventions«}

\section{François Eymard-Duvernay, Olivier Favereau, André Orléan, Robert Salais et Laurent Thévenot}

Traducteur : Oliver Ilan Schulz

\section{CpenEdition}

\section{Journals}

Édition électronique

URL : http://journals.openedition.org/trivium/3545

DOI : $10.4000 /$ trivium. 3545

ISSN : 1963-1820

Éditeur

Les éditions de la Maison des sciences de l'Homme

Référence électronique

François Eymard-Duvernay, Olivier Favereau, André Orléan, Robert Salais und Laurent Thévenot « Werte, Koordination und Rationalität: Die Verbindung dreier Themen durch die »Économie des conventions « », Trivium [Online], 5 | 2010, online erschienen am 10 Januar 2010, abgerufen am 08 September 2020. URL : http://journals.openedition.org/trivium/3545; DOI : https://doi.org/10.4000/ trivium.3545

Ce document a été généré automatiquement le 8 septembre 2020.

\section{c) (i) $\Theta$}

Les contenus des la revue Trivium sont mis à disposition selon les termes de la Licence Creative Commons Attribution - Pas d'Utilisation Commerciale - Pas de Modification 4.0 International. 


\title{
Werte, Koordination und Rationalität: Die Verbindung dreier Themen durch die »Économie des conventions«
}

\author{
François Eymard-Duvernay, Olivier Favereau, André Orléan, Robert Salais \\ et Laurent Thévenot \\ Traduction : Oliver Ilan Schulz
}

\section{NOTE DE L'ÉDITEUR}

Wir danken François Eymard-Duvernay und dem Verlag La Découverte für die freundliche Genehmigung, diesen Artikel zu übersetzen.

Das Forschungsprogramm der "Économie des conventions« (im Folgenden kurz: EC) führt aktuell drei Fragestellungen wieder zusammen, die das wirtschaftswissenschaftliche Denken anderthalb Jahrhunderte lang getrennt behandelt hat: die Charakterisierung der Akteure und ihrer Handlungsmotivationen, die Koordinationsmodalitäten von Handlungen sowie die Stellung von Werten und Gemeingütern. ${ }^{1}$ Bei der Erarbeitung der Standardtheorie wurden die Fragen der Rationalität und der Koordination strikt getrennt behandelt und getrennt axiomatisiert, erstere durch die Entscheidungstheorie, zweitere durch die Allgemeine Gleichgewichtstheorie. ${ }^{2}$ Diese beiden Fragen wurden wiederum von der dritten isoliert, die sich mit Werturteilen und normativen Überlegungen beschäftigt. Im Gegensatz dazu legen die von uns erarbeiteten Analyserahmen eine Verbindung dieser drei Fragen vor. Wenn man davon ausgeht, dass die Koordination menschlicher Handlungen problematisch ist und nicht etwa Naturgesetzen oder Zwängen folgt, kann man daraus schließen, dass die menschliche Rationalität zuallererst interpretativ und nicht nur oder zumindest nicht von vorneherein kalkulierend ist. Der Akteur muss zunächst mit Hilfe von konventionsbasierten Rahmen die Situation und das Handeln der anderen 
erfassen, um seine Handlungen koordinieren zu können. Dieses Erfassen ist nicht nur kognitiv, sondern auch evaluierend, wobei die Form der Evaluation über die Bedeutsamkeit und damit darüber entscheidet, was der Akteur erfasst und berücksichtigt. Hier erkennen wir den Stellenwert von kollektiven Werten und Gemeingütern in der Koordination, die nicht auf individuelle Präferenzen reduziert werden können, sondern die das Gerüst der legitimsten Konventionen für die Koordination bereitstellen. Als wichtiger Bestandteil von Institutionen findet hier auch die Sprache ihren Platz. Die EC strebt eine Integration an, die die Wirtschaftswissenschaften ebenso betrifft wie die Sozial- und Politikwissenschaften. Dieses Bestreben sollte also eher zu einer Annäherung beitragen als dass wir zusehen müssten, wie eine jede von ihnen versucht, sich auf Kosten der anderen auszudehnen.

2 Im ersten Teil gehen wir von der Feststellung aus, dass die Wirtschafts- und die Sozialwissenschaften sich gegenwärtig bekämpfen und versuchen, ihr Imperium auf den bevorzugten Bereich der Konkurrenzdisziplin auszuweiten. Wir teilen das Interesse für Verallgemeinerung. Sie offenbart aber ihre Grenzen, wenn die Erweiterungen einen Kern von Hypothesen beibehalten, der nicht alle Dimensionen koordinierten menschlichen Handelns integriert. In einem zweiten Teil kommen wir auf diesen Hypothesenkern zurück, um die von der EC bewirkten Verschiebungen aufzuzeigen. Die Ungewissheit, die über der Koordination schwebt, ist nicht mehr nur eine Frage der Informationsverteilung: Die Ungewissheit wird eingedämmt durch die interpretative Rationalität der Akteure und durch die Unterstützung, die die gemeinsamen Evaluationsrahmen erbringen, indem sie die relevanten Elemente der Situation bestimmen. Diese konventionsbasierten Koordinationsrahmen weisen vielfältige Formen auf, führen aber dennoch nicht zu einem Relativismus. Der dritte Teil arbeitet einen »horizontalen« Pluralismus von Qualifikationskonventionen heraus. Diese gehorchen ein und derselben Grammatik und weisen alle den höchsten Grad an Allgemeingültigkeit und Rechtfertigung auf. Dieser erste Pluralismus ermöglicht es, die Komplexität und Verschiedenartigkeit der Märkte sowie der wirtschaftlichen Organisationen zu analysieren und sie dabei nicht auf das Kalkül von Verträgen und Transaktionen zu reduzieren. Somit ist man besser gerüstet, um sich mit Unternehmen und den Besonderheiten der Arbeits- und Finanzmärkte zu befassen. Im vierten Teil führen wir einen zweiten, "vertikalen« Pluralismus ein, bei dem wir den Grad der Allgemeingültigkeit oder der Publizität der Koordinationskonventionen variieren lassen. Er ermöglicht eine Differenzierung des Oberbegriffs der Konvention, indem er lokalere Koordinationen und Informationsformate unterscheidet. Außerdem ermöglicht er es, die Analyse der politischen und moralischen Evaluationen zu verfeinern. Er gibt Auskunft über die Spannungen zwischen dem auf Äquivalenzen beruhenden Gerechten und den Bewertungen im Nahbereich. Letztlich wird der Übergang zwischen der Mikro- und der Makroebene auf diese Weise zwei Mal komplexer gestaltet. Ein erstes Mal durch die intrinsische Vielfalt der Verallgemeinerungsarten, und ein zweites Mal durch die Vertiefung des Verhältnisses zwischen dem Lokalen und dem Allgemeinen. 


\section{Auseinandersetzungen und Integrationsbemühungen der Wirtschafts- und Sozialwissenschaften}

3 Nehmen wir also den Ehrgeiz zur Verallgemeinerung ernst, der sich heute in der Wirtschaftswissenschaft wie in der Soziologie in Erweiterungsversuchen äußert, die sich auf den bevorzugten Bereich der jeweils anderen Disziplin erstrecken. Er führt zu einer interessanten Lage, in der es keine klare Revieraufteilung mehr gibt und in der es möglich wird, die Entwicklungen der sich überschneidenden Fortschritte der beiden Disziplinen gegenüberzustellen.

4 Ausgehend von einer Diagnose der auf beiden Seiten beobachteten Erweiterungen werden wir den Ansatz der EC rechtfertigen, der eine reflexive Betrachtung der beiden Disziplinen vornimmt. Im Gegensatz zu einem pluri- oder multidisziplinären Ansatz, der die Beiträge der unterschiedlichen Disziplinen einfach zusammenfügen würde, versucht unsere Perspektive, die Grenzen zwischen Wirtschaftswissenschaft und Soziologie zu überbrücken, um gemeinsame Grundlagen aufzudecken und diese in einem neuen Licht zu betrachten. Dennoch geht es nicht darum, die ursprünglichen Arbeiten der beiden Felder zu ignorieren oder sie zu vermischen. Die gemeinsamen Grundlagen der beiden Disziplinen neu zu überdenken ist umso dringlicher, als die Formen des Politischen (die Rolle des Staates und der intermediären Körperschaften; die Erzeugung des Allgemeinwohls; das individuelle Engagement für öffentliche Belange) heute stark vom Aufbau Europas und von der Suche nach internationalen Regeln als Antwort auf die Globalisierung beeinflusst werden. Da die Politikwissenschaft weitgehend von einer von der Wirtschaftswissenschaft entlehnten Thematik beherrscht ist (Governance, rationales Handeln, strategische Manipulation usw.), bietet sie keine angemessenen Mittel zur Rekonstruktion des Politischen.

\section{Von der Marktwirtschaft zu einer allgemeinen Wissenschaft der menschlichen Beziehungen}

5 Mit Dispositiven wie dem "Vertrag" oder dem "Spiel» dehnt sich die Wirtschaftswissenschaft auf nicht-marktbasierte Beziehungen aus. Diese Begriffe sind transaktioneller als das früher von der Allgemeinen Gleichgewichtstheorie dargebotene Bild und weichen damit von den ersten Erweiterungen (insbesondere im Sinne Beckers) ab. Die betroffenen Bereiche sind die Familie, die Staatsgewalt, die Politik, die Organisation; der Mechanismus des Markttausches ist nicht mehr der zentrale Mechanismus der Koordination. Es bleibt ein Begriff der allgemeinen Präferenz, die sich auf die Interaktionsmodalitäten mit dem Anderen ausdehnt. Dieser Präferenzbegriff, früher eng mit dem Raum der Güter und Dienstleistungen verbunden, gewinnt nun an Bedeutung, wie man besonders am Extremfall der Spieltheorie sieht. Die Waren werden durch Strategien ersetzt, auf die die Präferenzbeziehung Bezug nimmt.

6 Diese Erweiterung wirft Fragen auf. Sie will alle menschlichen Handlungsweisen abdecken und aus der Wirtschaftswissenschaft eine universelle Sozialwissenschaft machen, die die Gebiete aller anderen - der sozialen wie der politischen Gesellschaftswissenschaften besetzt. Aber wie kann dabei die Kohärenz mit dem ursprünglichen Kern der wirtschaftswissenschaftlichen Theorie und ihrem Anspruch auf Einheit bestehen bleiben? ${ }^{3}$ Die Verbindung mit dem Prinzip der Marktbeziehungen, 
aufrechterhalten durch die Bezugnahme auf Wettbewerb und Anreize, besteht darin, dass der Begriff "Vertrag", obwohl der Terminus nach vertraglichen juristischen Formen zu klingen scheint, vor allem eine Fortführung des Begriffs »Gleichgewicht« ist - eines Gleichgewichts zwischen Interessen, die nichts gemeinsam haben und sogar häufig antagonistisch sind. Um diese Fortführung zu ermöglichen, werden neue Gleichgewichtsbegriffe eingeführt (Nash).

In dem Bemühen, alle »Gleichgewichte« der Verhaltensweisen mit dem ursprünglichen Prinzip der konkurrenzbasierten Warenkoordination zu verbinden, öffnet sich die Erweiterung nur oberflächlich für eine Vielfalt vertraglicher Verfahren oder Transaktionsarten. Das beibehaltene Prinzip schließt in der Tat die Anerkennung einer interpretativen Rationalität ebenso aus wie die eines ethischen Urteils und einer Bezugnahme auf kollektive Objekte oder Wesen. Diese wären allerdings die grundlegenden Elemente einer Grammatik, wie sie der Pluralität der Koordinationsarten gemeinsam sind, die im Rahmen des Programms der Konventionen analysiert werden. Da eine solche Anerkennung fehlt, ist die Vielfalt der berücksichtigten vertraglichen Verfahren durch die Einheitsform des Gleichgewichts von Nash oder seiner Verfeinerungen eingeengt. Denn wie zahlreiche Autoren von Johansen bis Kreps festgestellt haben, besteht eine sehr enge Verbindung zwischen der Standard-Rationalität in einer Interaktionssituation und dem Konzept des Gleichgewichts von Nash.

\section{Die soziale Einbettung der Wirtschaft und die soziale Konstruktion der Märkte}

Die Wirtschaftssoziologie bietet gegenüber diesen Erweiterungen Alternativen an. Sie möchte die Wirtschaft auf einen Bereich reduzieren, der mit den anderen sozialen Handlungen, auf die sie sich spezialisiert hat, vergleichbar (homogen) ist. Der Vorteil dieser Erweiterung besteht darin, die als wirtschaftlich bezeichneten Beziehungen in einen viel größeren Raum zu übertragen und dabei ihre Verflechtung mit sozialen Handlungen hervorzuheben. In einem gemeinsamen Streben nach Denaturalisierung der Wirtschaftsbeziehungen ist ein reichhaltiger Komplex von Forschungsarbeiten über die »soziale Konstruktion der Märkte« entstanden.

9 Die Soziologie eröffnet den Zugang zu einer deutlich breiteren Palette sozialer Handlungen als dies die Spezifizierungen der wirtschaftswissenschaftlichen Theorie der Verträge zulassen (deren Beschränkung im Übrigen auf eine konzeptuelle Ökonomie abzielt). Somit ist die von der Soziologie vorgenommene Reduzierung, wenn sie sich auf den wirtschaftlichen Bereich ausdehnt, nicht so radikal wie die Reduzierung von Seiten der Wirtschaftswissenschaft. Für Soziologien interessegeleiteter oder strategischer Handlungen, die sich an Modellen ökonomischen Handelns orientieren, sind darüber hinaus manche Erweiterungen dadurch erleichtert, dass sie dabei bestimmte strategische Allianzen mit einer Wirtschaftswissenschaft nach Art Beckers eingehen können.

Diese Ausdehnung der Soziologie wirft jedoch vergleichbare Fragen auf, wie die Erweiterung des Bereichs der Wirtschaftswissenschaft. Selbst wenn die Modelle sozialen Handelns mehr oder weniger metaphorisch die Sprache der Märkte und des Nutzenkalküls übernehmen, setzen sie doch grundlegend andere Koordinationsformen voraus, weil sie sich auf soziale Gruppen stützen, auf soziale Repräsentationen, auf 
soziale Praktiken, auf einen sozialen Sinn und gegenseitiges soziales Verständnis. Sie schaffen es nicht, die Besonderheit der Handlungs- und Koordinationsrahmen $\mathrm{zu}$ beschreiben, an denen Waren beteiligt sind. Trotz seiner Ergiebigkeit zeugt der Begriff der "Einbettung" (embeddedness) des Wirtschaftlichen in das Soziale von dieser Reduktion auf Modelle sozialer Beziehungen.

\section{Eine nicht-reduzierende Integration}

Unser Projekt unterscheidet sich von diesen Erweiterungsbemühungen, die auf Hypothesen der Standard-Wirtschaftswissenschaften (dem Mainstream der Wirtschaftswissenschaften) oder der klassischen Soziologie beruhen. Es hat teil an den Integrationsbemühungen, von denen schon die beiden vorangehenden wissenschaftlichen Strömungen stimuliert werden. Aber es erkennt an, dass jede disziplinäre Tradition unterschiedliche Aspekte und Arten der Koordination erklärt, die von dem jeweils anderen fachlichen Rahmen bei seinem aktuellen Stand nicht erfasst werden können. Deshalb haben wir einen Analyserahmen konstruiert, der sich einer Frage verschreibt, die beiden Traditionen gemeinsam ist. Dieser Rahmen ermöglicht es uns, die Matrix $\mathrm{zu}$ bestimmen, die einer Pluralität von Koordinationsweisen zugrunde liegt und von der die verschiedenen fachspezifischen Hinterlassenschaften zeugen.

\section{Am Ausgangspunkt der Sozial- und Wirtschaftswissenschaften steht eine gemeinsame Fragestellung: die problematische Koordination der Handlungen}

\section{Eine gemeinsame Fragestellung für die Sozial- und Wirtschaftswissenschaften}

12 Die beiden vorangegangenen Formen der Erweiterung stoßen mit jenen Reduktionen an ihre Grenzen, die sie ausgehend von jeweils eigenen Begriffen der Handlung und der elementaren Koordination vornehmen, ganz gleich ob sie der wirtschafts- oder sozialwissenschaftlichen Disziplin angehören. Aber wenn wir uns auf grundlegendere Fragen zurückbesinnen, können wir eine gemeinsame Fragestellung von Soziologie und Wirtschaftswissenschaft erkennen: die problematische Koordination der menschlichen Verhaltensweisen. Jede Disziplin hat sich auf unterschiedliche Spezifizierungen dieser Koordination konzentriert. Wir möchten den daraus resultierenden Pluralismus bewahren, ohne ihn jedoch an die Fachgebietsgrenzen zurückzuführen oder uns auf einen Relativismus zu beschränken.

\section{Die Weiterentwicklung der Analyserahmen zur Erfassung des Ungewissheitscharakters der Koordinationen}

13 Welche Art von Akteuren und Dispositiven legt man zugrunde, um koordinierte Handlungen abzubilden? Die Antwort hängt natürlich davon ab, für welche Bedeutung des Worts Koordination man sich entscheidet. Wie wir schon in der Einleitung deutlich 
gemacht haben, hält sich die EC nicht an die Koordinationsdefinition der Wirtschaftswissenschaftler, die auf den methodologischen Individualismus zurückgeht, den sie in den neoklassischen Bewegungen oder in der Transaktionskostentheorie voraussetzen. Der von der EC entwickelte Koordinationsbegriff hebt die Stellung kollektiver Evaluationsformen hervor. Genauso wie die öffentlichsten Formen die Koordination einem Rechtfertigungsanspruch unterwerfen, setzen auch die Koordinationsarten von geringerer Tragweite Evaluationsformen voraus, die dann begrenzteren Gütern entsprechen. Darüber hinaus ist der so erweiterte Koordinationsbegriff mit der Idee des Konflikts vereinbar. Die Koordination ist eine Prüfung, die in Anbetracht von Versagen und insbesondere von Konflikt und Kritik ausgeführt wird.

Die »klassischen" Autoren der Wirtschaftswissenschaft und der Soziologie, die Referenzmodellen aus der Naturwissenschaft nahe stehen, haben wirtschaftliche und soziale Systeme entwickelt, die Gleichgewichte, Ordnungen und Strukturen der sozialen Reproduktion aufzeigen. Die Koordination wird bei ihnen systematisch von starken Kräften geleistet, die in den Akteuren und äußeren Zwängen angesiedelt sind. Neuere Ausführungen haben den Ungewissheitscharakter der Koordination herausgearbeitet, was voraussetzt, den Transaktions- und Interaktionsmodalitäten mehr Aufmerksamkeit zu widmen.

Für die interaktionistischen Soziologen bleibt die Ungewissheit in der Idee einer "Interaktionsordnung" enthalten, selbst wenn letztere lokal in der Situation "ausgehandelt" wird. Für die ethnomethodologischen Soziologen ist Ordnung besonders fragwürdig, und sie stellen sich damit den »Klassikern« entgegen. Aber im spezifischen Kontext der Situation bemühen sich die Akteure aktiv darum, durch »Ethnomethoden« um jeden Preis einen Common sense aufrechtzuerhalten. In einem größeren Zusammenhang gesehen erweitert der Begriff des wechselseitigen Verstehens die Idee einer sinnbasierten Einigung, der die Soziologen im Gegensatz zu den Wirtschaftswissenschaftlern große Bedeutung beimessen. Für die Soziologen der Akteur-Netzwerk-Theorie wird die Koordination erst im Nachhinein festgelegt und wird auf eine elementare Ebene der »Assoziation« und des »Enrolment« bezogen.

Für die Wirtschaftswissenschaftler konzentriert sich das Problem in den Begriffen Ungewissheit und Information. Die Standardtheorie hat, wenn auch erweitert um die Fragen der begrenzten Rationalität, ihr Handlungsmodell nicht infrage gestellt. ${ }^{4}$ Paradoxerweise bleibt die Unordnung sehr kalibriert und lässt zugleich mehr Raum für Opportunismus. Eine der grundlegenden Gewissheiten erklärt sich aus der Gegebenheit des Optionsraums, selbst wenn er auf den Warenraum übergreift ${ }^{5}$ und in der Spieltheorie ein Handlungsraum wird. Die Hypothese des common knowledge bleibt eine heroische, solange die Wege ihrer Entstehung und ihrer Einhaltung in der Koordination nicht untersucht worden sind. ${ }^{6}$ Das setzt voraus, dass man die materiellen, sozialen und institutionellen Bedingungen ernst nimmt, die den sich koordinierenden Personen ihr Engagement bei der Handlung ermöglichen. Das setzt auch voraus, die Black Box des rationalen Handelns zu öffnen, um dort Reflexivität und Vernunft zu suchen. Das alles sind Perspektiven, denen sich die Wirtschaftswissenschaft trotz der wiederholten Appelle ihrer großen Autoren weitgehend verweigert. 


\section{Wie kann man es besser machen? Die Ungewissheit über eine Evaluationsform beschreiben, die die Koordination unterstützt}

die die Koordination der Verhaltensweisen beeinflusst, ganz gleich ob diese Ungewissheit aus einer Asymmetrie an Informationen oder aus dem besonderen Kontext einer Situation entsteht. Was machen wir besser? Wir unterscheiden Formen der Ungewissheit und damit auch der Information und verknüpfen sie mit unterschiedlichen Evaluationsformen, denn schließlich steht die Evaluation im Zentrum der Koordination.

Mit den Begriffen »Unvollständigkeit« oder »radikaler« bzw. »kritischer « Ungewissheit versuchen wir unterhalb der Formung jener Information anzusetzen, auf die sich das Kalkül der Wirtschaftswissenschaftler der Vertragstheorie stützt. Wir unterscheiden die Prozesse, mit denen der Zweifel kanalisiert und mit verschiedenen Wissens- und Informationsformaten bearbeitet wird, deren Relevanz wiederum einem Koordinationsmodus zugeordnet wird. So können wir verstehen, warum nichtmarktförmige Koordinationsformen andere Wissensformate verlangen, wohingegen diese Formen gewöhnlich von den Wirtschaftswissenschaftlern auf Informationsungleichheit reduziert werden, die sich beispielsweise auf die wesentlichen Eigenschaften von Waren und Dienstleistungen bezieht.

Anfangs gibt es in keiner Koordination eine Ordnungsmäßigkeit, die als sichere Stütze gelten kann, ganz gleich, ob die Koordination auf dem Markt oder im Unternehmen stattfindet oder auf eine politische Einigung abzielt. In diesem Sinne ist die Ungewissheit für jeden (auch für den Theoretiker, der den Lauf der Dinge verstehen und ihn nicht nur von außen und im Nachhinein erklären will) radikal. In ihrer alltäglichen Besonderheit ist jede Koordination in dem Maße ungewiss, als sie heterogene Akteure ins Spiel bringt, einen zeitlichen Ablauf hat und auf ein Produkt (oder eine Dienstleistung) abzielt, das nie gänzlich vordefiniert ist.

Diese Ungewissheit gilt es durch die konventionsbasierte Konstruktion von Produkten, Dienstleistungen und Antizipationen zu überwinden, die die Grundlagen für den Warenaustausch und die Produktionstätigkeit der Unternehmen sind. ${ }^{7}$ Der Konventionsbegriff ermöglicht es, diesen Moment der gemeinsamen Konstruktion zu beschreiben. Ungewissheit zu überwinden ist wohlgemerkt das tägliche Brot des Lebens in Gemeinschaft und, in weiterem Sinne, der Gesellschaft. Beobachtungen zeigen, dass dies den Akteuren oft gelingt, zumindest bis zu einem gewissen Grad. Sie legen nahe, dass diese Konventionskonstruktion eine permanente, individuelle und kollektive Tätigkeit darstellt, die in das Handeln integriert ist.

21 Ausgehend von einer gemeinsamen Evaluationsform, die die Objekte für die Koordination qualifiziert, kanalisieren die Konventionen die Ungewissheit. Wir werden also von der Marktqualifikationskonvention im eingeschränkten Sinne eines wettbewerbsorientierten Konsumgütermarkts andere Qualifikationskonventionen unterscheiden, die es uns um den Preis eines harten Bruchs mit dem vorherrschenden Paradigma ermöglichen, eine breitere Palette an Transaktionen abzudecken, und damit den aktuellen Bestrebungen der Wirtschafts- und Sozialwissenschaften entsprechen. ${ }^{8}$ Die Anerkennung einer Pluralität solcher Konventionen reagiert damit auf die Kritik bezüglich einer ungerechtfertigten Erweiterung des Markts auf die Gesamtheit der Interaktionen, ohne die nicht-marktbasierten Beziehungen auf einen »sozialen 
Rahmen« des Marktes zu übertragen. Jenseits der Pluralität der Koordinationsarten und der daraus entstehenden Brüche muss man also von einem gemeinsamen Rahmen für diese unterschiedlichen Koordinationsarten ausgehen, weil sonst der Wechsel von einer zur anderen, wie ihn die Akteure durchlaufen müssen, nicht verstanden werden kann.

Die Ungewissheit zu erfassen und die Konventionen zu spezifizieren, die die Grundlage der Qualifikation der Transaktionsobjekte sind, führen zu der Erkenntnis, dass die Relevanz eines Wissensformats von einer Evaluationsform abhängt. ${ }^{9}$ Die Evaluation steht im Zentrum der Koordination und ist weder ein Argument unter vielen für die individuelle Nutzenfunktion - ein unsichtbarer Sockel, der an andere Disziplinen weitergereicht wird -, noch ein Wert, der der Rationalität beigefügt wird, um sie zu vervollständigen oder zu korrigieren.

\section{Der erste Pluralismus der allgemeinen Koordinationsarten: Welche Art der Legitimität, welche Art der Integration?}

Legitime Evaluationsformen stärken die Institutionen. Wenn man anerkennt, dass sich die allgemeinsten Koordinationsarten auf solche Formen stützen, führt das dazu, dass man die Ansprüche auf Gerechtigkeit und Demokratie ernst nimmt, die an diese Organisationen gestellt werden. Dasselbe gilt für den Sinn für Gerechtigkeit, Gemeingut und öffentliches Gut, den man von den Akteuren erwartet, die in diese Koordinationen eingebunden sind. Der Stellenwert dieser Ansprüche, die im Zentrum der politischen Philosophie stehen, hat in den gängigsten wirtschaftswissenschaftlichen und soziologischen Ansätzen beträchtlich abgenommen. Entweder reduzieren diese Ansätze jede Evaluation auf individuelle Präferenzen, die in einen Preis integriert werden, oder sie verkürzen sie auf soziale Werte, die in ihrer Vielfältigkeit beliebig sind. Nimmt man die Legitimität dieser Evaluationsformen und deren Pluralismus ernst, werden sowohl die Akteure als auch die Organisationen anders wahrgenommen werden.

\section{Politik, Gerechtigkeit, Demokratie}

Wenn man einen Pluralismus der legitimen Koordinationsarten anerkannt hat, gelingt es dann auch, diese in das Regierungssystem von Organisationen oder Staaten $\mathrm{zu}$ integrieren? Können wir den Relativismus vermeiden, der in den Wirtschafts- und Sozialwissenschaften gewöhnlich mit der Pluralität der Werte verbunden ist?

Die Analyse der Beziehungen zwischen den legitimsten Koordinationsarten und dem Sinn für Gerechtigkeit bzw. Ungerechtigkeit kommt zu einer positiven Antwort. Anstatt uns mit einer Wertetypologie oder Weberschen Idealtypen wie den unterschiedlichen Herrschaftsarten $\mathrm{zu}$ begnügen, haben wir gezeigt, dass verschiedene Qualifikationsordnungen, durch die allgemeine Koordinationsarten ihre Legitimität gewinnen, einer selben Grammatik der Gerechtigkeit bzw. der Ungerechtigkeit gehorchen. Die Evaluationsformen, die zu einer Beurteilung führenden Prüfungsverfahren und die Beziehungen zwischen Evaluationsordnungen werden dann als ungerecht angeprangert, wenn sie nicht einer Gesamtheit von Voraussetzungen genügen. Diese sind in einem Modell offen gelegt worden, dem eine Pluralität von 
Rangordnungen nach Wertigkeiten (grandeurs, 》Größen«) gemein ist. ${ }^{10}$ Zwischen diesen Voraussetzungen und zwei aktuellen Theorien der Gerechtigkeit bzw. der Ungerechtigkeit haben wir gewisse Übereinstimmungen festgestellt: einerseits mit dem zweiten Prinzip der Gerechtigkeitstheorie von Rawls, andererseits mit dem Pascalschen Verständnis der Tyrannei im Sinne einer Herrschaft außerhalb ihrer eigenen Ordnung. Letztere beeinflusste die Unterscheidung der Gerechtigkeitssphären durch Walzer und diente darüber hinaus als Matrix für die Analyse der Rangordnungen nach Größen. Allerdings führt der pragmatische Einstieg über die Koordination der Handlungen und nicht direkt über die Güterverteilung dazu, dass wir uns von diesen Autoren entfernen. Er ermöglicht es, die Verfahren der Koordinationsprüfung und ihre Fundierung auf qualifizierte Objekte zu verdeutlichen, ebenso wie die Beziehung zwischen den Verfahren und den substantiellen Gütern, die oft nicht beachtet werden - insbesondere nicht beim Gegensatz zwischen gerecht und gut, der durch die liberalen Grammatiken radikalisiert wird.

Indem wir Koordinationsdispositive ausgehend von diesen Gerechtigkeitsgrammatiken analysieren, können wir deren demokratische Öffnung und die Grenzen dieser Öffnung einschätzen. Diese Einschätzung beschränkt sich nicht auf den Gegensatz zwischen Staat und Zivilgesellschaft, sondern behandelt so vielfältige Orte wie den Staat, öffentliche Maßnahmen, die von Verbänden übernommen wurden, Normierungsgremien, Regulierungsbehörden, Konferenzen und Abstimmungsforen etc. ${ }^{11}$ Die staatsbürgerliche Ordnung macht einen Anspruch verständlich, der für jede demokratische Politik unerlässlich ist, denn sie bestimmt eine Suche nach Gleichheit und Solidarität und stützt sich dabei auf rechtlich ausgewiesene Regelwerke. Die klare Abgrenzung von einer Marktordnung ermöglicht es, Irrtümer zu vermeiden, die aus den möglichen Kompromissen zwischen den liberalen Politikgrammatiken und der Konvention des Marktes entstehen könnten - Kompromisse, die durch die Erweiterung einer übermäßig auf Marktbeziehungen fokussierten Wirtschaftswissenschaft in den Bereich des Politischen häufig verdeckt werden.

\section{Die Akteure und ihre unterschiedlichen Zustände beim Bewerten}

Im wirtschaftswissenschaftlichen Modell ist die Evaluation durch die Akteure in der Nutzenfunktion konzentriert, die als stabil oder nur exogenen Variationen unterworfen gilt. Mehrere Forschungsrichtungen versuchen die Präferenzen $\mathrm{zu}$ endogenen umzudeuten, entweder, indem sie sie mit Routinen gleichsetzen, die durch das Umfeld ausgewählt werden, oder indem sie eine Rangordnung der Präferenzen einführen, die Metapräferenzen.

Alle diese Forschungsansätze haben ihre Vorzüge. Wir versuchen aber, darüber hinauszugehen, und verbinden die Evaluation mit einem Zustand der Personen, der von ihrem Involviertsein (Engagement) innerhalb ihrer Koordinationsumgebung abhängt. So ordnen wir die routinierten Verhaltensweisen nicht nur einfachen, reflexionslosen Regelmäßigkeiten zu, sondern einem Modus des Involviertseins, bei dem die Gewöhnung an ein vertrautes Umfeld bewirkt, dass sich die Bewertung auf einer lokalen Anpassungsebene vollzieht. Andererseits verknüpfen wir den ethischen Inhalt der Metapräferenzen mit einem Involviertsein ganz anderer Dimension, bei dem die kollektive Grundlage der Evaluation entscheidend ist. 
Gegensatz zur verbreiteten Standardtheorie, die das Koordinations- oder Kooperationsproblem dadurch zu erfassen versucht, dass sie das Kosten-Nutzen-Kalkül des homo oeconomicus auf die Ebene des individuellen Ichs beschränkt, haben Sozialpsychologen (Tajfel, Turner) die komplementäre Rolle zweier weiterer Ebenen aufgezeigt, nämlich der des sozialen Ichs (durch Gruppenzugehörigkeiten) und der des Ichs als menschliche Person. Diese Hierarchie ermöglicht es, Wechsel von einer Ebene auf die andere zu verstehen.

Im Vergleich zu den Soziologien, die für das Sozialverhalten stabile Determinanten voraussetzen, berücksichtigt die EC eine Pluralität von Evaluationszuständen, ermöglicht damit unterschiedliche Formen des Involviertseins und macht die Dispositionen der Personen beweglicher. Zudem werden dadurch die dispositionalen Bewegungen mit den Veränderungen der Dispositive in Beziehung gesetzt, in denen die Handlungen stattfinden und die externe Träger der Evaluation enthalten. Eine derartige Analyse bewahrt die Möglichkeit, die Beziehungen zwischen diesen Dispositionen und den sozialen Zugehörigkeiten zu untersuchen, aber sie lässt ihre Bewegung zu, so wie man sie bei Mitgliedern von Gesellschaften beobachten kann, in denen jedes mit verschiedenen Einschätzungsarten umgehen muss.

\section{Die konstitutiven Konventionen des Marktes und der Unternehmen ...}

Märkte sind zuallererst Orte, an denen die Qualität der Güter geprüft und evaluiert wird. Ihre Organisation unterliegt den Aktivitäten, die diese Prüfung vorbereiten und in eine Form gießen: Kodifizierungs-, Mess-, Zertifizierungs- und Regulierungsaktivitäten usw. Es sind Aktivitäten, die den Märkten vor- oder nachgeschaltet und dennoch mit ihnen verbunden sind. ${ }^{12}$ Der Staat ist dabei als Empfehlender oder als Bürge präsent.

Der Zustand von Personen als Akteure auf einem Markt hat die Wirtschaftswissenschaftler am meisten beschäftigt. Man muss feststellen, dass es bedauerlich ist, das Geschehen auf einem Markt auf die Gesetze von Angebot und Nachfrage zu reduzieren. Die Akteure werden einzig als Käufer und Verkäufer identifiziert, obwohl doch »hinter« dieser Identifizierung diejenige als Konsument und Produzent mindestens genauso bedeutsam ist. Konsumenten und Produzenten richten konventionsbasierte Erwartungen an das Tauschobjekt, die nicht einfach durch mechanische Anpassung von Angebot und Nachfrage zur Deckung kommen. Jeder hat ein wesentliches Vorrecht. Die Konsumenten bestimmen souverän über die Qualität ihres Einkaufs und ihre Kaufentscheidungen. Die Produzenten entscheiden souverän über die Fabrikationsregeln der Produkte und Dienstleistungen. Diese beiden Vorrechte bilden den Ausgangspunkt der Einigungen über die Qualität der Güter auf dem Markt, ja sie machen sie überhaupt erst möglich. Die tatsächliche Konkurrenz in jedem Markttyp hängt vom Prüfungs- und Evaluierungstyp ab, der sich auf diesem Markt durchsetzt. Die Evaluationsverfahren unterscheiden sich je nach Markt; sie sind je nach Art der Transaktionsobjekte verschieden: Produkte und Dienstleistungen vielfältiger Art und Bestimmung, Arbeit, Finanztitel. Wie wir im Weiteren sehen werden, gibt es auf allen diesen Märkten eine Pluralität von Evaluationsprinzipien, die in die theoretische Analyse integriert werden muss. 
Die kollektive Form des Zustands der Personen, ihre Qualifikation, ist durch die Koordinationszwänge bedingt. Zur Entstehung einer gemeinsamen Evaluation bedarf es eines Verfahrens, um die individuellen Evaluationen zusammenzuführen. Anders als die übereifrigen Kritiker des Marktes annehmen, ist der Konsument auf dem Markt kein unabhängiges Individuum, das von allen Bindungen frei ist: Zur Gesamtheit der Marktgüter hat er nur Zugang, wenn er zahlungsfähig ist und sich in den Zustand des Konsumenten versetzt, was ihm gewisse Rechte und Pflichten verleiht. Er muss sich insbesondere an das Güterangebot und an die Marktpreise halten. Unter diesen Voraussetzungen kann eine gefestigte Nachfrage entstehen. Man stößt hier auf ähnliche Zwänge wie bei der Auszählung einer politischen Abstimmung. Der Konsumentenzustand stützt sich auf Dispositive, insbesondere auf Handelsgüter, die ein Wissensformat in die Beziehungen einführen. Lokalere Ansätze betonen Verhaltensweisen, die von diesem allgemeinen Zustand abweichen: über Preise kann diskutiert werden, an den Objekten der Transaktion können Anpassungen vorgenommen werden etc. Sie führen zu einem zweiten Pluralismus, den wir im folgenden Abschnitt vorstellen.

\section{... und die der anderen legitimen Koordinationsarten}

34 Wir können die anderen Koordinationsarten einführen, wenn wir den Zustand der Akteure variieren, der deren Evaluation dessen bestimmt, was ein Gut ist. Der Terminus "Gut« ist in der Wirtschaftswissenschaft ganz offensichtlich mehrdeutig, denn er kann ebenso sehr die angeeignete Sache wie auch das bezeichnen, was allgemeiner gesprochen eine Evaluation steuert. Das ist nicht nur eine Quelle für Missverständnisse, denn wir versuchen, das Gut einer Transaktion mit den pluralen Möglichkeiten einer Evaluation zu verbinden, die nicht auf die Marktevaluation beschränkt sind. Für diesen pluralistischen Ansatz ist der Begriff Gut sehr offen und ermöglicht es, sich vom Marktgut $\mathrm{zu}$ entfernen. Die klassische Unterscheidung zwischen Gut und Dienstleistung, die vom erweiterten Rahmen des Marktes eingeschränkt worden ist, hat eine tiefere Bedeutung: Sie suggeriert bereits Zustände des Transaktionsobjekts, die zu verschiedenen Wissens- und Evaluationsformen führen. Der erweiterte Begriff des Guts kann also eine so breite Palette von Koordinationsarten abdecken, wie sie in der Wirtschaft und der modernen Gesellschaft erkannt werden, ohne sie auf eine einzige Form zu begrenzen.

Es gibt vielfältige Strategien zur Einführung von Koordinationen, die sich vom Markt unterscheiden. Ihnen allen ist gemeinsam, dass sie Evaluationszustände definieren, die von dem des Konsumenten abweichen. ${ }^{13}$ Auf diese Art können wir solche Produktionsaktivitäten und Arbeit besser analysieren, die Güterevaluationen ins Spiel bringen, deren Wissensformat nichts mit dem des Konsumenten zu tun hat. Für den Wirtschaftswissenschaftler ist die Produktionsfunktion die Art und Weise, diese Formen der Koordination in einem Modell darzustellen. Um die Souveränität des Marktes zu erhalten, reduziert er sie dabei aber auf technische Zwänge. In der Debatte über den Arbeitswert und den Gebrauchswert ist diese Spannung zwischen verschiedenen Koordinationen schon seit den Ursprüngen der Wirtschaftswissenschaft spürbar. Mit dem Interesse für die Unternehmensanalyse gewinnt sie gegenwärtig wieder an Aktualität, aber die Vertragstheorie kann sie nicht richtig erfassen. Es ist 
bezeichnend, dass Herbert Simon ein alternatives Paradigma der Rationalität entwickelt und sich dabei in erster Linie mit Organisationen beschäftigt hat.

Hat man den Pluralismus der Evaluationen erst einmal erkannt, nimmt man die Grenzen der Informationsökonomie umso deutlicher wahr: Die Informationsasymmetrie zwischen den Akteuren ist meistens ein Verteilungsproblem der Evaluationsfähigkeiten und des Evaluationsmodus. So kann man von Informationsasymmetrie sprechen, wenn man unterstreichen will, dass der Arzt in seiner Beziehung zum Patienten über mehr Informationen verfügt als der Kranke: Er könnte das ausnutzen, um ihn zu täuschen, und sich beispielsweise bei der Heilung eines alten Mannes weniger Mühe geben, obwohl dieser denselben Preis für die Dienstleistung bezahlt wie ein anderer Patient. Das erklärt sich aus einer Evaluationsfähigkeit, die - wenn sie dem Mediziner vorbehalten ist - zu einem Machtmissbrauch führt, weil der Kranke nicht an diesem Evaluationsmodus hinsichtlich der Wirksamkeit der Behandlungsformen beteiligt ist. Die positive Seite der Asymmetrie, die aus dieser Fähigkeit herrührt, wird im ausschließlich negativen Ansatz der Verträge ignoriert. Die Pluralität der Evaluationsformen wird auf eine geordnete Asymmetrie reduziert. Damit vernachlässigt die Informationsökonomie die fraglos entscheidende Erzeugung von Informationsformaten (Wissens- und Evaluationskategorien), die relevant werden und zukünftig als gemeinsames Wissen gelten.

Die Pluralität der Koordinationen stimmt nicht mit den Grenzlinien organisierter oder etablierter Aktivitätskategorien überein. Eine gegebene wirtschaftliche Aktivität kann, selbst wenn sie in kleine Tranchen aufgeteilt ist, zu mehreren Koordinationsformen gehören, dasselbe gilt für Unternehmen. In derartigen pluralistischen Kontexten besteht das Koordinationsproblem im Zusammentreffen von mehreren Evaluationsprinzipien sowie in der Verteilung der Evaluationsmacht auf verschiedene Zustände der Personen.

Um diese Pluralität in einen gemeinsamen Analyserahmen zu integrieren, muss man sich erneut mit der Frage des Gleichgewichts beschäftigen. Das Gleichgewicht zwischen Angebot und Nachfrage hat es der wirtschaftswissenschaftlichen Theorie ermöglicht, das Marktmodell $\mathrm{zu}$ erweitern. Danach hat das Nash-Gleichgewicht eine neue Erweiterung geboten. Diese Gleichgewichte beruhen auf Akteuren und Transaktionsobjekten, die zunächst in den Zustand des Marktes versetzt worden sind, oder in den Zustand eines Pseudo-Vertragsmarkts. Wenn wir das »Gleichgewicht« in einem pluralistischen Rahmen beibehalten wollen, bezieht sich der Begriff auf die Stabilität dieses Zustands, der eine Bedingung für die konjunkturbedingte Regulierung des Preises ist (oder anderer Orientierungspunkte für die Koordination). Ein Ungleichgewicht entsteht, wenn die Evaluationsprinzipien, die den Zustand der Personen und Dinge bilden, in Frage gestellt werden, insbesondere, wenn man sich dabei auf alternative Koordinationen stützt. In einem pluralistischen Rahmen stellt die von $\mathrm{H}$. White vorgenommene Modellierung der Produktmärkte einen besonders stimulierenden Weg der Formalisierung dieses erneuerten Gleichgewichtsbegriffs dar. ${ }^{14}$ Das Gleichgewicht, das heißt also die Fortführung der Qualitätskonvention, bezieht sich folglich eher auf eine Dispersion als auf einen Mittelwert. 


\section{Die Bewegung der konstitutiven Konventionen} die Gründungsinstitutionen (der Markt, die Kollektivität) für exogen, universell und stabil zu halten. Die Einführung in die Analyse der radikalen Ungewissheiten (Fehlen eines Koordinationsmodus, der die Ungewissheit integriert, indem er sie näher bestimmt) und der kritischen Dynamiken (Infragestellung einer Einigung) führt $\mathrm{zu}$ einer Konzeption von pluralen und sich entwickelnden Konventionen, die durch Handlungen verformt werden (Salais et al., 1998). Die Personen befinden sich in einer konventionsbasierten Umgebung (die insbesondere aus Texten, juristischen Korpora, Berechnungseinheiten und Evaluationsinstrumenten gebildet ist), die sie neu gestalten, um die Koordinations- und Kooperationsmängel $\mathrm{zu}$ beheben. Um diese Konventionsdynamik in die Analyse einzuführen, muss man die Akteure mit einem reflexiven Verhalten bezüglich ihres Zustands versehen, und mit der Fähigkeit, die Formen ihres Zusammenlebens neu zu gestalten, also der Fähigkeit zur Politik.

Die Versuche, die politischen Verhaltensweisen ausgehend von der Rationalität einzuführen, sind zum Scheitern verurteilt: Die Vertragstheorie hat sich zwar für einen solchen Ansatz entschieden, aber das Gleichgewicht wird nur um den Preis einer unrealistischen Rationalitätshypothese und eines im Hintergrund aufrecht erhaltenen allgemeinen Marktes gewahrt. Neben dem rationalen Verhalten ein ethisches und altruistisches Verhalten einzuführen ermöglicht es nicht, den pluralen und evolutionären Charakter der Güter zu erfassen, die bei der Einschätzung der Verhaltensweisen eine Rolle spielen. Man muss den politischen Anspruch in die Analyse integrieren, indem man ihn der Konventionsdynamik und der Art des Referenzguts zuordnet.

\section{Seltsame Märkte: der Arbeitsmarkt und der Finanzmarkt}

41 Seit Langem schon kritisieren die institutionalistischen Strömungen die Erweiterung und die Vereinheitlichung, die der Begriff des Marktes angeblich ermöglicht. Zur Kritik von Polanyi an einem Kommodifizierungsprozess kamen die Kritiken an der neoklassischen undifferenzierten Behandlung aller Märkte hinzu. Das Prinzipal-AgentModell würde nichts Gutes aus dem Verhalten eines Personalchefs schließen. North und Williamson sind sich dieser Grenzen der Erweiterung bewusst, aber sie begnügen sich damit, das Politische wie eine Instanz zu behandeln, die willkommene Anreize liefert. Da sie den ökonomischen Akteur nur in seinem »individuellen« Zustand betrachten, sind sie gezwungen, das Politische in einem Register zu entwerfen, das dem Menschen jegliche politische oder auch nur soziale Fähigkeit abspricht. Die EC ermöglicht es, weiter zu gehen, und akzeptiert die theoretische Spezifizität aller institutionellen Marktdispositive. Damit knüpft sie an die Tradition der Klassiker an.

\section{Die Arbeitsmärkte}

Die marktbasierte Reduktion der Arbeit auf einen von den Konsumenten bestimmten Produktionsfaktor verfälscht die gemeinsame Wahrnehmung der Evaluationen, die an der Arbeit festgemacht sind. ${ }^{15}$ Für das Marktmodell ist die Arbeit eine Art negativer Konsum, die nur dazu dient, Kaufkraft zu liefern. Der Arbeiter ist also im Zustand des 
Konsumenten, der sich zwischen Güterkörben entscheidet, in denen auch die Freizeit integriert ist. Daher rührt die Fokussierung auf »Drückeberger«-Verhalten, um die Arbeitstätigkeit zu beschreiben. Die Geschicklichkeit und Methode, die eingesetzt, und die Anstrengungen, die aufgewendet werden, um eine berufliche Tätigkeit durchzuführen, werden so auf eine Unnützlichkeit (Désutilité) reduziert. Daraus ein altruistisches Verhalten zu machen ist nur eine wenig befriedigende Umkehrung desselben Modells. Eine bessere Lösung besteht in der Einführung eines Zustands, der mit der Arbeitstätigkeit verbunden ist, und der auf einer spezifischen Konzeption des Guts der geschickten Tätigkeit basiert. Anstatt ausschließlich über die Entlohnung zu gehen und damit die isolierte Betrachtung eines Arbeitsmarktes zu ermöglichen, findet die Finalität der Arbeit auch durch das Gut einer Aktivität statt, die mit einem Produkt konsolidiert wird, dessen Wert den der Arbeit indexiert. Dieser Ansatz ist offen für die Pluralität der Arbeitsformen. ${ }^{16} \mathrm{Er}$ ermöglicht es, die Spannungen zwischen unterschiedlichen Evaluationen des Guts anhand der Evaluationen des Produkts zu analysieren.

Die Stellung der Unternehmen wird neu gefasst. In der Fortführung der Transaktionskostenökonomie wird das Unternehmen als ein Koordinationsrahmen betrachtet, der sich vom Markt unterscheidet. Nach Coase oder Williamson hat die Firma nur auf die Effizienz der Koordination Einfluss und setzt über die Transaktionen und die Verträge die neoklassische Tradition einer allgemeinen Reduktion auf den Warenaustausch fort. Unserer Ansicht nach organisiert das Unternehmen die Verbindung zwischen den Güter-, Arbeits- und Kapitalmärkten. Es müssen auch größere Koordinationsräume als das Unternehmen eingeführt werden, zum Beispiel die Branche, wenn die Regeln der Güter- und Arbeitsaufwertung aus diesem Rahmen hervorgehen.

Darüber hinaus steht das Unternehmen am Schnittpunkt mehrerer Koordinationsformen und bewältigt die Spannungen, die in solchen Situation entstehen, durch Kompromisse zwischen diesen Koordinationsformen. Die Vielfalt der Unternehmensmodelle und der Produktionswelten, auf die die Analyse der Koordinationskonventionen hinausläuft, stellt die Sichtweise in Frage, die das Unternehmen als einheitlichen und einfach hierarchischen Koordinationsmodus betrachtet. ${ }^{17}$

\section{Die Finanzmärkte}

Eine Aktie zu besitzen verleiht ein Recht auf den zukünftigen Fluss der erwarteten Dividenden. Es handelt sich lediglich um ein Versprechen auf Geld. Für den Besitzer folgt daraus ein Risiko, denn er kann - wenn er sich mit unvorhergesehenen Ausgaben konfrontiert sieht - in große Schwierigkeiten geraten, weil er seine Aktien nicht sofort in Geld umwandeln kann. Die Wertpapierbörsen sind institutionelle Gründungen, die erfunden wurden, um eine spezifische Forderung von Gläubigern zu erfüllen: Eigentumsrechte flüssig zu machen. Mit dieser Aussage behaupten wir das genaue Gegenteil der gängigen Finanzanalyse. Denn diese betrachtet die Wertpapiere gleich Waren als natürlich austauschbar. Alle Bemühungen der EC zielen darauf ab, diesen Naturzustand zu kritisieren, in dem Güter für den Austausch bereitstehen. Die Analyse der Finanzmärkte zeigt deren Unterschiede hinsichtlich zweier Evaluations- und Koordinationsarten auf, mit denen sie leider oft verwechselt werden. ${ }^{18}$ 
Zunächst zeigt sich die Abkoppelung von der industriellen Welt der produktiven Investitionen in der Tatsache, dass der Preis des Wertpapiers nicht Ausdruck eines »inneren Werts « im Sinne der Fundamentalanalyse ist. Die finanzielle Liquidität leitet einen radikalen Schnitt ein zwischen der Zeit der Produktion und der Zeit des Finanzwesens. Während die Erschließung des Produktivkapitals ein langwieriger Prozess ist, weil sie eine unumkehrbare Festlegung des Kapitals erfordert, produziert die Liquidität ununterbrochen Möglichkeiten der Neubewertung und damit des Gewinns. Dieser Unterschied des Zeitzustands, den man schon im Zentrum der Kapitalismusanalyse von Keynes vorfindet, zeigt unserer Meinung nach deutlich die Diskrepanz zwischen der Evaluation auf den Finanzmärkten und der Evaluation eines Produktivkapitals, das einer Qualifikationskonvention industrieller Art entspricht.

Dennoch lassen sich die Finanzmärkte nicht auf eine konkurrenzfähige Koordinationsart reduzieren, die ebenso auf eine Marktqualifikation der Güter gegründet wäre wie andere Konsumgütermärkte. Das Finanzwesen bedingt eine Koordination durch Meinungen, die eine Gesamtheit heterogener Meinungen in einen von allen akzeptierten Referenzwert verwandelt. Die Antizipationen der Akteure sind auf die Antizipationen der anderen Beteiligten gerichtet. Dadurch kommen mimetische Verhaltensweisen zur Geltung, und diese Nachahmung auf individueller Ebene führt zu sehr schädlichen Situationen für die Wirtschaft, wie beispielsweise der Entstehung von Spekulationsblasen, das heißt dauerhaften Diskrepanzen zwischen den Börsenkursen und den »inneren Werten«.

Bei einer Koordination, die auf einer durch Meinung generierten Qualifikationskonvention beruht, bestimmt der Zeichencharakter - also das wieder erkennbare Hervorstechende - die Dinge und erzeugt das dieser Koordinationsart zugehörige »Objektivitäts«-Format. Solange das Konventionsobjekt akzeptiert ist, ist die Spekulationsdynamik beträchtlich vereinfacht, denn um vorauszusehen, was die anderen machen werden, muss man sich nur an die Konvention halten. Durch das Spiel der Selbstbestätigung der Überzeugungen erlangt die Konvention eine relative Stabilität und wird für die Akteure so etwas wie eine zweite Natur.

\section{Der zweite Pluralismus der Ebenen der Konventionen: Von der öffentlichen Ebene bis in den Nahbereich}

Die Untersuchung von offiziellsten Institutionen und staatlichen Politikprogrammen, aber auch von Organisationen, Unternehmen oder Vereinigungen, deren demokratische Ansprüche wir berücksichtigen wollen, hat dazu geführt, dass wir uns zuerst mit den legitimsten Koordinationsarten beschäftigt haben. Dennoch kann unsere Analyse sich nicht mit dieser Ebene begnügen, die in den Evaluationen und Qualifikationen der Personen und Dinge nur auf einen öffentlichen Legitimitätsimperativ abzielt. Unser Forschungsprogramm hat sich also einem zweiten Pluralismus geöffnet, um situative Koordinationen sowie persönlichere Formen des Involviertseins in den eigenen Nahbereich (Gewohnheiten und Gepflogenheiten convenances) aufzugreifen. Ohne uns auf die kognitiven Aspekte so genannter »stillschweigender« oder »informeller« Kenntnisse zu beschränken, haben wir uns mit den Evaluationen und Gütern befasst, die an diesen lokaleren Koordinationen beteiligt sind. 
In Soziologie und Wirtschaftswissenschaft haben sich verschiedene Strömungen mit Handlungsmodalitäten befasst, die unterhalb der Forderungen nach Beratung und öffentlicher Kritik, ja sogar unterhalb des individuellen Nachdenkens liegen. In Habitus, Routinen und Praktiken haben sie unreflektierte Beziehungen zur Welt ausgemacht, die auf Inkorporation beruhen und kontextabhängig sind, zumindest was ihr Erlernen angeht. Als die Hypothese der begrenzten Rationalität die Hypothesen über das rationale Kalkül und die vollständigen Kenntnisse des Akteurs gelockert hat, trug sie zugleich dazu bei, der jeweiligen Situation einer Handlung mehr Gewicht zu verleihen. Das Interesse für den Kontext und die Konzeptionen einer situativen Handlung haben die Aufmerksamkeit von der Beratung, der Auswahl zwischen geregelten Optionen oder der Planung in Richtung einer Berücksichtigung der Umstände verschoben. Die Netzwerkanalysen untersuchen die Umstände auf ihre eigene Art und gehen dabei von einer Vielzahl von Verbindungen aus. Dennoch haben sich diese Entwicklungen auf die kognitiven Organisationen dieser Nahbeziehungen konzentriert, ohne dabei die beteiligten Evaluationen und Güter zu berücksichtigen. Diese Theorien laufen Gefahr, sich zu sehr auf lokale Aktionsmodelle zu konzentrieren, dadurch die Koordinationsansprüche größerer Tragweite zu disqualifizieren und die weitergehende Verallgemeinerung zu verfehlen, den die Öffentlichkeit und die Politik einfordern. Dies trifft deutlich auf die evolutionistischen Modelle von Routineverhalten $\mathrm{zu}$, die aus einer Modellbildung von »lokalen« Bindungen hervorgehen. Auch die Vertragsmodelle bleiben partiell und interindividuell, da die Verbindung zum restlichen Markt auf willkürliche und exogene Weise durch die Bedingung der so genannten Beteiligung hergestellt wird. Die wirtschaftswissenschaftliche Theorie bietet uns also zwei lokale Modelle an, das eine mit einer schwachen Rationalität (Routinen), das andere mit einer starken Rationalität (Verträge) - beide sind nicht zufriedenstellend.

Umgekehrt blenden die zu sehr auf den öffentlichen Raum, die Institutionen oder die Staatsbürgerschaft konzentrierten Theorien die Prämisse einer Person aus, die sich durch Nähe aufrechterhält. Sie erkennen die Vielfalt der Handlungsformen nicht und können die Bewegungen nicht erfassen, die notwendig sind, um von einer dieser Formen zur anderen zu wechseln: so wenn eine Regel oder ein Recht »konkrete Anwendung erfährt«, öffentliche Politik den Menschen "nahegebracht« werden soll oder ein funktionelles Objekt oder ein Plan einem spezifischen Gebrauch angepasst werden. Umgekehrt verlangt der Weg zu öffentlichen Qualifikationen Zustandsänderungen von Dingen, aber auch von Personen, die sich vom Nahen freimachen müssen - um die Autonomie zu gewinnen, für ein Projekt verantwortlich zu sein, eine Meinung zu unterstützen oder um an eine öffentliche Qualifikation $z u$ gelangen. Das Nahe ist nicht nur das Besondere des Allgemeinen, es beruht auch auf spezifischen Arten des Involviertseins in Situationen. Die Bewertungen, die sich auf Formen des Involviertseins im Nahbereich stützen, bereichern nicht nur die berücksichtigten Wissensformen, sondern auch die Evaluationen und die Urteile über das Ungerechte, den Machtmissbrauch und alle Arten von Unrecht, das Personen zugefügt wird. Deshalb hat sich unser Forschungsprogramm in Richtung einer Differenzierung von Handlungs- und Koordinationsformen entwickelt, die dazu dienen soll, die Wechsel von einer Form zur anderen zu verstehen und den Missbrauch aufzudecken, der aus der Vorherrschaft der einen über die anderen entsteht. Ein Programm, das schon auf den Pluralismus der legitimsten Koordinationsarten achtet, muss auch auf einen zweiten Pluralismus eingehen, der der unterschiedlichen 
Tragweite der koordinierten Handlungsregimes geschuldet ist, die vom öffentlichen bis in die nächste Nähe reichen. ${ }^{19}$

\section{Die Reduktionen der Handlungen und Interaktionen im Nahbereich in der Wirtschaftswissenschaft}

Handlungen und Interaktionen im Nahbereich werden von den Wirtschaftswissenschaftlern häufig eher stiefmütterlich behandelt. Die Überlegungen zu den Asymmetrien oder Unvollständigkeiten von Informationen beschäftigen sich eigentlich mit Situationen, die unter dem Gesichtspunkt der von den unterschiedlichen Akteuren verwendeten Informations- und Evaluationsformate asymmetrisch sind, ebenso wie der Gegensatz zwischen zentralisierter und dezentralisierter Information. Mehrere Forschungsströmungen versuchen innerhalb der Vertragstheorie die Beziehungen des Nahbereichs zu formalisieren, die von den kanonischen Modellen, die sich auf die substantielle Rationalität der Akteure gründen, schlecht erfasst werden. Die Modelle unvollständiger Verträge weisen in diese Richtung. Da sie die Hypothese der substantiellen Rationalität aber nicht grundlegend revidieren, fallen sie wieder auf den Standardansatz zurück. Für eine zufriedenstellendere Lösung müsste man die Verwurzelung des Wissens in Dispositiven anerkennen, die die Spuren der Interaktionen mit dem Umfeld bewahren, sowie die Stellung der Evaluationen.

Die Evolutionisten machen bei ihren Überlegungen $\mathrm{zu}$ Arbeit und Produktionsorganisationen das Routinemodell gegenüber dem Planmodell stark. Auf diese Weise soll der reflexionslose Charakter der Tätigkeit und deren Abhängigkeit von der Vergangenheit betont werden. Da die Akteure in einem komplexen Umfeld nicht sorgfältig abwägen können, greifen sie auf frühere Gewohnheiten zurück, und die Koordination beruht auf diesen Routinehandlungen. Trotz dieser Beiträge ist der Nachteil dieses Ansatzes, dass er sowohl die höheren Ebenen vernachlässigt, die für den Sinn des Legitimen erforderlich sind, wie auch die niedrigere Ebene der persönlichen Gewöhnung. Denn die Routine wird nach Art der sozialen Gepflogenheiten oder der Bräuche meistens wie eine regelmäßige und häufig kollektive Gewohnheit behandelt. Das Spezifische des persönlichen Involviertseins im Vertrauten wird ebenso wenig berücksichtigt, wie die Schwierigkeiten, die daraus für die Koordination mit anderen Personen entstehen, die mit diesem Vertrauten nicht vertraut sind. Bei der Frage des Lernens stößt man aber durchaus auf solche Schwierigkeiten.

Ebenso wie die effektiven Arbeits- und Produktionsaktivitäten Zusammenhänge implizieren, zu denen die Produktionsfunktionen und Vertragsformalismen im Allgemeinen keine Aussagen machen, beschränkt sich die reale Nutzung der Produkte und Dienstleistungen weder auf den funktionalen Umgang mit Dingen, von dem die Nutzenfunktion zeugt, noch erschöpft sie sich in der Zerstörung durch Konsum. In der wirtschaftswissenschaftlichen Literatur finden sich spärliche Hinweise auf ein Nutzungsregime, das die Art der fortschreitenden und spezifischen Anpassung einer Person an seine Umgebung festlegt. So betont der Begriff "Erfahrungsgut" die Abhängigkeit von einer Erfahrung, anstatt sich mit einem Konsumverhältnis zu begnügen. Aber mit der Reduktion dieses Nutzungsregimes auf die Eigenschaften einer Ware geht die Charakterisierung einer Handlungsweise verloren, die damit einhergeht. Auch die path-dependence-Modelle gestehen den kontingenten Besonderheiten des 
Milieus zwar einen Einfluss auf den späteren Verlauf zu, aber nur, um sie auf suboptimale Entscheidungen bei der Wahl der technischen Mittel zurückzuführen.

\section{Von regelbasierten Institutionen zu Interaktionen}

Die Koordination von Handlungen ist nicht gleichmäßig etabliert. Fußen die Institutionen, wie in der Einleitung des dritten Abschnitts gezeigt, auf den legitimsten Konventionen, so weichen doch viele Handlungen vom institutionellen Format ab und wählen andere, für den Nahbereich geeignetere Formate, auch wenn sie mit den Institutionen verbunden bleiben. Man erkennt das heute daran, wie öffentliche Politikprogramme sich an lokalen Gegebenheiten anpassen und so nah wie möglich an die Personen und Situationen herankommen wollen.

Unter den Konventionen von maximaler kollektiver Tragweite kann man zunächst die konstitutiven Konventionen ersten Rangs (Konvention $\left.{ }_{1}\right)_{\text {unterscheiden. Sie }}$ unterstützen die legitimsten Koordinationsarten und sind folglich von sehr großer Bedeutung hinsichtlich der Urteile und der gemeinsamen Güter, auf denen die Evaluationen gründen. Sie sind mehr als nur Regeln, die die Koordination als normal erachteter Handlungen ermöglichen. Ihr Interpretationsspielraum entspricht dem der Rechtfertigung und der Kritik, der dem Anspruch auf demokratische Debatte zu eigen ist. Im Gegensatz dazu umfassen Konventionen zweiten Rangs (Konvention ${ }_{2}$ ) begrenztere Regeln, die dazu bestimmt sind, normierte Handlungspläne $\mathrm{zu}$ koordinieren. ${ }^{20}$ Sie lassen weniger Interpretationsspielraum. Dieser findet seine Grenzen im Verhältnis zur Regel des richtigen Handelns.

Die Analyse von Institutionen oder öffentlichen Politikprogrammen zeigt, dass es Aktivitäten gibt, die nicht auf diese Konventionen größter kollektiver Tragweite reduziert werden können, nämlich Handlungen von Akteuren, die zu den öffentlichen Organismen gehören und sich an die »Nutzer« annähern wollen, oder Evaluationssituationen, in denen der Evaluierende eine direkte Interaktion mit der evaluierten Person eingeht. ${ }^{21}$ Diese Handlungen drücken sich in der alltäglichen und nicht formalisierten Sprache der Erzählung aus, es fehlt die von den Institutionen geforderte Orthodoxie. Handlungen, Absichten und objekte werden alltagssprachlich benannt, indem man sich eines Formats bedient, das gegenüber den institutionellen Formen einen Spielraum erlaubt. Die Koordination zwischen den Akteuren ist keiner Forderung nach stärkerer Verallgemeinerung unterworfen, die die Übereinstimmung mit der Institution sicherstellt, sondern vollzieht sich durch Interaktionen, in denen die etablierten Prüfungen erleichtert oder sogar ausgesetzt sind, und das zugunsten von Leistungen, die in einem toleranteren Format der entsprechenden Handlung bewertet werden.

Mit Blick auf die etablierte Handlung wird diese Form der Interaktion häufig nur negativ als eine »informelle« oder »lokale« betrachtet. Wir erwägen eher ein Pendeln zwischen den verschiedenen Koordinationsebenen, das die positiven Seiten dieser Annäherung von Personen betont. Eine solche dynamische Perspektive muss sich vor zwei Reduktionen hüten, die in Bezug auf Institutionen weit verbreitet sind: die der holistischen Konzeption, die sie als kollektive Strukturen darstellt, die strikt alle sozialen Praktiken bestimmt, und die der individualistischen Konzeption, die die Institution auf eine Anhäufung individueller, interessegeleiteter Handlungen verkürzt. Diese beiden Optionen schränken die Palette der Bewertungsformen erheblich ein, die 
die Personen dabei leiten, ihr eigenes Verhalten oder das der anderen zu erfassen. Die Berücksichtigung des Rechts setzt voraus, dass man auch seine laufenden Verfahren betrachtet, und zwar so, dass man es in einer Differenzierung der Ebenen verortet, die es nicht nur auf den strengen Wortsinn reduziert.

Aufgrund des kognitiven Zwangs zur Verallgemeinerung und des politischen Zwangs zu einer Gleichbehandlung aller Akteure durch die Institution mobilisieren die institutionellen Regeln konstruktionsbedingt allgemeine Kategorien zur Erstellung von Äquivalenzen. Darüber hinaus setzen sie eine Evaluation eines gemeinsamen Guts von großer Bedeutung voraus, bei der die Akteure sich zu »Gesetzgebern« machen und eine kritische Haltung zu der Frage einnehmen, was eine gute Regel sei. Bezüglich dieses Urteils zeigen die Ebene der Interaktion in einer bestimmten Situation und das Pendeln, das sie mit formalisierteren Koordinationen erlaubt, vier Typen von Weiterführungen.

Erstens kann die Evaluation mit allgemeinen Kategorien brechen, die Vorurteile fördern, und zu einem individualisierten Urteil übergehen, das eine Abfolge von Handlungen des Individuums berücksichtigt. Auf einem Niveau unterhalb der Formalität des Diploms kann dieses Urteil die Kompetenzen des Individuums bewerten, von denen seine Taten zeugen und die nicht Gegenstand eines förmlichen staatlichen Akts waren. Etablierte Kategorien wie Diplome reichen nicht aus, um eine gerechte Evaluation zu garantieren, und die Berücksichtigung von Handlungskompetenzen, die sich während der Interaktion zeigen, kann dank der Abschwächung der von den etablierten Kategorien herbeigeführten Vorurteile zu einer gerechteren Behandlung führen.

61 Zweitens wird die Evaluation durch in der Situation verfolgte Zielsetzungen vollendet, die sie in eine eingeschränktere Planung einfassen als das Streben nach einem Gemeingut. Wenn das angestrebte Gut über den Rahmen einer gut ausgeführten Handlung hinausgeht, kann es lokal und unterhalb des Strebens nach Universalisierung bleiben. So werden die Unternehmen durch Dispositive getragen, die sich zumeist mit lediglich lokalen Koordinationsanforderungen zufrieden geben, während sich das angestrebte Gut auf das Unternehmen beschränkt, ohne sich auf die gesamte Gesellschaft auszudehnen.

Drittens kann sich die Evaluation für die Pluralität der legitimen Rechtfertigungsprinzipien öffnen. Bei den lokalen, von kombinierten Dispositiven gestützten Gütern gehen diese Prinzipien im Übrigen oft Kompromisse ein. Eine derartige Öffnung sorgt für Unerwartetes, weil sie die Situation unter einem neuen Blickwinkel erscheinen lässt. Ein Urteil kann als »ausgeglichen« bezeichnet werden, wenn es sich nach den durch diese Prinzipienwechsel herbeigeführten Variationen stabilisiert, und nicht, wenn die Situation vorher unter Berücksichtigung eines einzigen Prinzips bereinigt wurde.

63 Viertens kann die Evaluation in der Interaktion einen Dialog auslösen, der zu einer Verringerung der Asymmetrien zwischen dem Evaluierenden und dem Evaluierten führen und den Schwächeren dadurch zugute kommen kann, dass darin ihre Rechte berücksichtigt werden. Man kann in diesem Fall von einem "ausgehandelten« Urteil sprechen und die Ansicht vertreten, dass es den Ausdruck von Ungerechtigkeiten begünstigt, die bisher nicht zur Kritik vorgedrungen waren. Dieses Interaktionsregime darf also nicht auf eine Beschädigung der Gerechtigkeit reduziert werden - mit der Begründung, dass die Gleichheit durch die Auflösung der allgemeinen Kategorien 
geschwächt würde und die Objektivität des Urteils durch die Pluralität der Prinzipien beeinträchtigt. Es bietet vielmehr Voraussetzungen für eine vielfältigere Beurteilung der Personen.

Zum Schluss sei noch angemerkt, dass diese situative Form der Interaktion in einer vielfältigen Palette von Institutionen stattfindet - den Markt eingeschlossen, wenn die Evaluation der Güter dort über die vorgefassten Meinungen hinausgeht, die auf die allgemeinen Kategorien begründet sind.

\section{Auf der Ebene unterhalb des individuellen Subjekts: das Involviertsein im Vertrauten als Halt der Person}

Wenn staatliche Politikprogramme in Sachen Rückführung in die Arbeitswelt, Wiedereingliederung, Wohnungsraum oder, allgemeiner, sozialer Arbeit angepasst werden, damit sie bürgernäher werden, appellieren sie an individuelle Entwürfe, Projekte, an Vorsätze und Willensbekundungen, die sich jeweils beweisen müssen. Sie haben dabei den Zustand eines Individuums im Blick, das in der Lage ist, sich in seiner Planung zu koordinieren und einen eigenständigen Willen und eigene Meinungen $\mathrm{zu}$ äußern. Dieser Appell konfrontiert die betroffenen Personen mit einer Forderung, die als Voraussetzung für den Zugang zu einem öffentlicheren Koordinationsniveau gilt und das wiederum auf den legitimsten Konventionen beruht. Wenn also der Zustand des Individuums die Grundlage von Verpflichtungen, von Sich-Einbringen im öffentlichen Raum bildet, dann entspricht das in der Erfüllung der passenden Handlungen schon einem Konsolidierungsniveau der Person, ganz wie wir es im vorangehenden Abschnitt festgestellt haben.

Allerdings zeigt die Erfahrung der Akteure dieser staatlichen Politikprogramme, dass vielfach gegen diese Forderung nach individueller Autonomie verstoßen wird. Zumeist verweist man auf einen Komplex von Unzulänglichkeiten: Mangel an Willenskraft oder Beharrlichkeit, Passivität oder Untätigkeit, Unfähigkeit, seine Zusagen einzuhalten. Viele Wirtschaftswissenschaftler sehen darin eine Entscheidung für die Untätigkeit. Im Gegensatz dazu betonen die Soziologen soziale Faktoren und Determinanten, die die Personen der Verantwortung derartiger Unzulänglichkeiten entheben.

Beide Ansätze verkennen, dass die Person, vor ihrem Zustand als autonomes Individuum, an Anknüpfungspunkten aus dem Nahbereich Halt finden muss, die sie im Vertrauten binden. Die verschiedenen personalisierten Betreuungsmaßnahmen, die die staatlichen Politikprogramme fortführen, beruhen auf solch einem der Person Halt gebenden Vertrautheitsverhältnis unterhalb des Zustands des aktiven Individuums. Die Dynamik der persönlichen Gewohnheiten und Gepflogenheiten (convenances personelles) beruht auf aus dem Gebrauch heraus aufgetretenen Orientierungspunkten im Nahbereich, und zwar während des Umgangs mit einer Umgebung, in der die Person unterkommt. Hier verweilt sie bevorzugt, gehalten durch ihre Bindungen. Gewöhnlich nehmen die Sozialwissenschaften diese Dynamik der Anpassungen im Vertrauten nur verzerrt wahr - in der Alternative aktiv/passiv, im schlechten Ansehen einer die Autonomie des Subjekts beeinträchtigenden Abhängigkeit, im versteiften und repetitiven Begriff Routine oder in einer Entstellung, die diese persönlichen Konvenienzen als kollektiv gewordener Brauch und Kultur zeigt. Die politischen Konstruktionen können dieses grundlegende Gut, das in einem Vertrauten, in dem die Person wurzelt, eingebunden ist, nicht ignorieren - denn schließlich geht es ihnen um 
Würde, versprechen sie mit Blick auf die Unterschiede zwischen den Personen eine gastlichere gemeinsame Welt und bemühen sie sich um Formen der Anerkennung dieser Unterschiede und der Bekämpfung der Diskriminierungen, die sie hervorrufen.

\section{Fazit}

Am Schluss dieses Parcours, der mit der Charakterisierung eines zweiten, "vertikalen« Pluralismus endet, welcher die eher lokalen Formen des Involviertseins von den öffentlicheren Konventionen unterscheidet, stoßen wir wieder an die Grenzen der wirtschaftswissenschaftlichen Standardtheorie. Doch nun verfügen wir über die Mittel, die Erweiterungen, die der Ausgangspunkt dieses Texts waren, in einem neuen Licht zu betrachten. Im Gegensatz $\mathrm{zu}$ den Sozialwissenschaften, die wegen ihrer Empfänglichkeit für kollektive Wesen kritisiert werden, traut sich der Vertreter einer erweiterten Standardtheorie zu, sich allen menschlichen Handlungen anzunähern, inklusive derer, bei denen in anderen Disziplinen auf soziale Kollektive oder politische Gemeinschaften zurückgegriffen wird, indem er sich letztlich auf das beschränkt, was er für den elementarsten und realistischsten Zustand des Menschen hält: den des interessegeleiteten Individuums. In unserer Konstruktion dagegen bezieht das Individuum in seinem Verhalten ein normatives Streben nach Koordination mit den anderen und dem Gemeingut ein, statt sich hinter einem egoistischen Kalkül zu verschanzen. Darüber hinaus können wir jetzt erkennen, dass diese Individualität, die aus der Person vor allem ein Zentrum für Entscheidungen und des Kalküls macht, weder der Urzustand noch die Basis aller menschlichen Koordinationen ist. Dieses Format eines autonomen Individuums ist nur auf der Grundlage einer Persönlichkeit zugänglich, die in den vielfältigen Formen des Involviertseins im Vertrauten ihren Halt findet. Werden sie durch eine zerstückelte Tätigkeit oder Wohnsituation zerstört, können sie der Person sogar ihre Privatsphäre rauben. Verständlich werden nun die zwei Schwachpunkte in den Erweiterungen der Standardtheorie, wenn sie sich mit Sozialpolitik und insbesondere der Wiedereingliederungspolitik beschäftigen: Sie verkennen in der Koordination mit den anderen die Bezugnahme auf das Gemeingut, im vorliegenden Fall ein staatsbürgerliches Gut der Solidarität; den Zustand des Individuums, den die Wiedereingliederungspolitik eben gerade wieder herstellen will, setzen sie als gegeben voraus.

\section{BIBLIOGRAPHIE}

Benetti, C. / Cartelier, J. (1980): Marchands, salariat et capitalistes, Paris, Maspero.

Boltanski, L. / Thévenot, L. (2007 [1991]): Über die Rechtfertigung. Eine Soziologie der kritischen Urteilskraft. Aus dem Französischen von Andreas Pfeuffer, Hamburg, Hamburger Edition.

Dupuy, J.-P. (1989): »Conventions et common knowledge«, Revue économique, 2, S. 361-400.

Dupuy, J.-P. et al. (1989): „L'économie des conventions«, Revue économique, 2, März. 
Eymard-Duvernay, F. (1989): „Conventions de qualité et formes de coordination«, Revue économique, 2, S. 329-359.

Eymard-Duvernay, F. (2002): »Conventionalist approaches to enterprise«, in: Favereau, O. / Lazega, E. (Hg.): Conventions and Structures in Economic Organization: Markets, Networks and Hierarchies, Cheltenham, Edwar Elgar, S. 60-78.

Eymard-Duvernay, F. (2004): Économie politique de l'entreprise, Paris, La Découverte.

Eymard-Duvernay, F. / Marchal, E. (1994): »Les règles en action: entre une organisation et ses usagers«, Revue française de sociologie, 35, S. 5-36.

Eymard-Duvernay, F. / Marchal, E. (1997): Façons de recruter. Le jugement des compétences sur le marché du travail, Paris, Métailié.

Favereau, O. (1986): »La formalisation du rôle des conventions dans l'allocation des ressources«, in: Salais, R. / Thévenot, L. (Hg.): Le Travail: marchés, règles, conventions, Paris, Economica, S. 249268.

Favereau, O. (1997): »Economics and its models«, in: d'Autume, A. / Cartelier, J. (Hg.), Is Economics Becoming a Hard Science?, Cheltenham, Edwar Elgar, S. 120-146.

Favereau, O. (2001): »Theory of information: from bounded rationality to interpretative reason«, in: Petit, P. (Hg.): Economics and Information, Dordrecht, Kluwer, S. 93-120.

Favereau, O. / Biencourt, O. / Eymard-Duvernay, F. (2002): »Where do markets come from? From (quality) conventions! «, in: Favereau, O. / Lazega, E. (Hg.): Conventions and Structures in Economic Organization: Markets, Networks and Hierarchies, Cheltenham, Edwar Elgar, S. 213-252.

Orléan, A. (1999): Le Pouvoir de la finance, Paris, Odile Jacob.

Orléan, A. (Hg.) (2004 [1994]): Analyse économique des conventions, 2. Aufl., Paris, PUF.

Salais, R. (1989): „L'analyse économique des conventions du travail«, Revue économique, 2, S. 199240.

Salais, R. / Chatel, E. / Rivaud-Danset, D. (Hg.) (1998): Institutions et conventions. La réflexivité de l'action économique, Paris, Éd. de l'EHESS.

Salais, R. / Strorper, M. (1993): Le Mondes de production, Paris, Éd. de l'EHESS.

Salais, R. / Thévenot, L. (Hg.) (1986): Le Travail: marchés, règles, conventions, Paris, INSEEEconomica.

Thévenot, L. (1997): »Un gouvernement par les normes; pratiques et politiques des formats d'information«, in: Conein, B. / Thévenot, L. (Hg.): Cognition et information en société, Paris, Éd. de l'EHESS, S. 205-241.

Thévenot, L. (2002): „Conventions of co-ordination and the framing of uncertainty«, in: Fullbrook, E. (Hg.): Intersubjectivity in Economics, London, Routledge, S. 181-197.

Thévenot, L. (2006): L'action au pluriel, Paris, La Découverte.

\section{NOTES}

1. Dupuy et al (1989); Orléan (1994); Salais / Thévenot (1986).

2. Favereau (1997).

3. Favereau (1997). 
4. Favereau (2001).

5. Hypothèse de nomenclature: Benetti / Cartelier (1980).

6. Dupuy (1989).

7. Eymard-Duvernay (1989); Salais / Storper (1993).

8. Eymard-Duvernay (2004).

9. Thévenot (2002)

10. Boltanski / Thévenot (2007 [1991]).

11. Thévenot (1997).

12. Favereau / Biencourt / Eymard-Duvernay (2002).

13. Eymard-Duvernay (2004).

14. Favereau / Biencourt / Eymard-Duvernay (2002).

15. Salais (1989).

16. Eymard-Duvernay (2004).

17. Eymard-Duvernay (2002).

18. Orléan (1999).

19. Thévenot (2006).

20. Favereau (1986).

21. Eymard-Duvernay / Marchal (1994), (1997).

\section{INDEX}

Schlüsselwörter : Handlungskoordination, Ökonomie der Konventionen, Ungewissheit Mots-clés : coordination des actions, économie des conventions, incertitude

\section{AUTEURS}

\section{FRANÇOIS EYMARD-DUVERNAY}

François Eymard-Duvernay ist Professor für Wirtschaftswissenschaft an der Universität Paris Ouest Nanterre La Défense. Nähere Informationen finden Sie hier. 